\title{
Conversion of a Constant Phase Element to an Equivalent Capacitor
}

\author{
Byoung-Yong Chang* \\ Department of Chemistry, Pukyong National University, 45 Yongso-ro, Nam-gu, Busan 48513, Republic of Korea
}

\begin{abstract}
Here I present a formula which converts a constant phase element (CPE) to its equivalent capacitor. Electrochemical impedance spectroscopy is capable of resolving a complex electrochemical processes into its faradaic and non-faradaic elements, and the non-faradaic process is frequently described as a CPE in place of a capacitor due to the non-ideality. Being described as a capacitor, the non-faradaic element provides information by its capacitance, but a CPE cannot provide a physical meaning. In order to solve the problem, the CPE has been dealt with as an equivalent capacitor of which the capacitance provides practical information. Succeeding the two methods previously suggested, a new conversion method is suggested in this report. While the previous ones manipulate only the CPE, the new method takes both the CPE and its related resistor into account for conversion. By comparing the results obtained by the three methods, we learn that the results are nearly the same within tolerable ranges, and conclude that any of the method choices is acceptable depending on the conditions of the system of interest.
\end{abstract}

Keywords : Electrochemical Impedance, Constant Phase Element, Nyquist Plot

Received : 19 February 2020, Accepted : 8 April 2020

\section{Introduction}

Electrochemical impedance spectroscopy is the only electrochemical measurement technique which can resolve electrochemical processes into faradaic and non-faradaic components. Generally, an electrochemical process consisting of a faradaic and a nonfaradaic components is described as an equivalent circuit consisting of a resistor and a capacitor connected parallelly [1]. It is obvious that a faradaic process can be characterized as a resistor because charges are transferred directly during the process, but it is vague to designate a non-faradaic process to a specific component. When an electrode is polarized, charges are accumulated at the electrode/solution interface of which the medium is dielectric material to drive formation of an electric double layer. As a result of that, a non-faradaic process is mostly designed as a capacitor. Not only that, adsorp-

*E-mail address: bychang@pknu.ac.kr

DOI: https://doi.org/10.33961/jecst.2020.00815

This is an open-access article distributed under the terms of the Creative Commons Attribution Non-Commercial License (http://creativecommons.org/licenses/by-nc/4.0) which permits unrestricted non-commercial use, distribution, and reproduction in any medium, provided the original work is properly cited. tion of charged molecules in the electrolyte medium and a delayed response of a faradaic reaction also show capacitive effects [2-4].

Even though such a non-faradaic component is expected to be presented as an ideal capacitor, the practical observation results in non-ideal behaviors of a capacitor; while an ideal capacitor shifts electric signals by the phase of $\pi / 2$, the practical signals are measured being shifted by $n \cdot \pi / 2$ where $n$ is given between around 0.8 to 1 . Owing to the phase shift by a certain degree, the element is called a constant phase element (CPE) that also includes a normal capacitor when $n=1$, and its impedance is expressed below [5],

$$
Z_{C P E}=\frac{1}{Y_{0}(i \omega)^{n}}
$$

where $\omega$ is angular frequency, $Y_{0}$ and $n$ are the characteristic parameters of the CPE. Therefore, instead of a capacitor, a CPE is very frequently used in analysis of electrochemical impedance spectrum in many practical cases [6-9].

A CPE definitely results in a better fitting curve than a capacitor, but its parameter, $Y_{0}$, does not give 
clear physical meaning as capacitance does. Over this problem, E. van Westing suggested a formula to convert $Y_{0}$ to an equivalent capacitance [10].

$$
C=\frac{Y_{0}(\omega)^{n-1}}{\sin (n \pi / 2)}
$$

Then, many scientists proposed practical applications of the above formula to real systems. Again, C. H. Hsu and F. Mansfeld suggested an advanced conversion method in their technical note [11].

$$
C=Y_{0}\left(\omega_{\max }^{\prime \prime}\right)^{n-1}
$$

with $\omega_{\max }^{\prime \prime}$ is the frequency where the imaginary part of impedance has its maximum.

Here I present another conversion method developed from a different approach. The two well-known method try to calculate the capacitance only using the imaginary part of impedance, but the new method uses both the imaginary and real parts of impedance interfered by the CPE. As a result, we can find the effective capacitance and resistance for the parallel circuit consisting of a constant phase element and a resistor.

\section{Theory and Simulations}

In order to find an equivalent capacitance of a CPE, the circuit of a resistor $\left(R_{\mathrm{p}}\right)$ and a CPE in Fig. 1(a) needs to be modified to another equivalent circuit consisting of a resistor and a capacitor in parallel. The conversion methods of eq (2) and (3) are based on the assumption that the CPE only affects the imaginary part of impedance, and build the equivalent circuit in Fig. 1(b). However, as the impedance of a CPE in eq (1) is rearranged to $\frac{1}{Y_{0} \omega^{-n}}\left(\cos \left(\frac{n \pi}{2}\right)-i \sin \left(\frac{n \pi}{2}\right)\right)$, the real part of impedance should be accounted in the modified circuit. Therefore, we can set up another circuit of which the CPE and the $R \mathrm{p}$ are replaced with a $C_{\text {eff }}$ and a $R_{\text {eff }}$ as shown in Fig. 1(c).

It is already known that a CPE can also be described by distribution of time constants and has electrical impedance expressed by the equation below [12],

$$
Z=\frac{R_{o}}{\left[1+(i \omega T)^{n}\right]}
$$

where $T$ is the time constant for the decaying current and $R_{0}$ is a resistor. Here, we can infer that the decaying current is rendered by the capacitive element which is parallelly connected to a resistor, which leads to the distributed time constant to be defined as

$$
T=C_{e f f} \cdot R_{e f f}
$$

As the impedance of the circuit in Fig. 1(a) is expressed below

$$
Z=\frac{R_{p}}{\left[1+(i \omega)^{n} Y_{0} R_{p}\right]}
$$

and should yield the same impedance results of eq (4), the following equation is derived.

$$
T=\left(Y_{0} R_{p}\right)^{1 / n}=C_{e f f} \cdot R_{e f f}
$$

Fig. 2 shows Nyquist plots for the circuit in Fig. 1(a) with $n=0.8(\mathrm{a}, \bullet)$ and $1\left(\mathrm{~b},-{ }^{-}\right)$. Plot (b) shows the same semi-circle of the Nyquist plot of the circuit in Fig. 1(b) having an equivalent capacitance converted from $Y_{0}$ by eq (2) or (3). While Plot (b) with $n$ $=1$ shows a perfect semi-circle, Plot (a) with $n=0.8$ does a depressed semi-circle. Even though the semicircle of Plot (a) looks depressed, it should be a perfect semi-circle if the circuit has a capacitor and resistor in parallel. So, the circuit of Fig. 1(a) can be re-designed to that of Fig. 1(c). The perfect semi-circle can be found when it is made with (a)

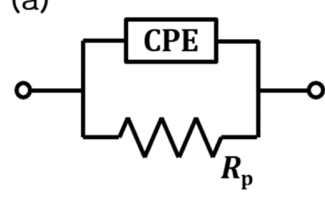

(b)

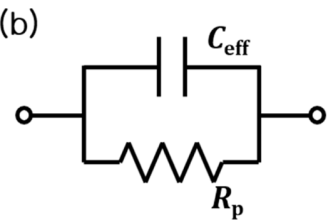

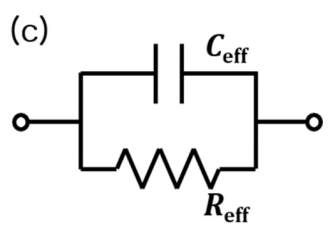

Fig. 1. (a) A circuit composed of a resistor $\left(R_{\mathrm{p}}\right)$ and a constant phase element (CPE) in parallel to describe a faradaic and a non-faradaic processes. (b) A circuit equivalent to circuit (a) with the CPE replaced by an effective capacitor. (c) A circuit equivalent to circuit (a) with the $\mathrm{CPE}$ and $R_{\mathrm{p}}$ replaced by an effective capacitor and an effective resistor, respectively. 


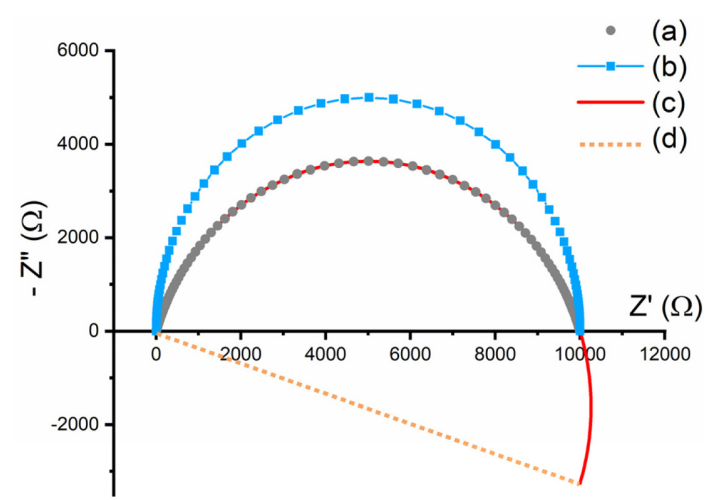

Fig. 2. Nyquist plots of the circuit in Fig. 1(a) calculated with $R_{\mathrm{p}}=1 \times 10^{4} \Omega, Y_{0}=1 \times 10^{-5} \mathrm{~s}^{n} / \Omega$ with $n=0.8(\mathrm{a}, \bullet)$ and 1 (b, - - ), respectively. Plot (b) is the same as the Nyquist plot of the circuit in Fig. 1(b). Plot (c, -) is a semi-circle obtained with appropriate $R_{\text {eff }}$ and $C_{\text {eff }}$ with a phase angle of $n \pi / 2$ and includes Plot (a). Line (d, --) is the diameter of the semi-circle of Plot (c).

$$
R_{\text {eff }}=R_{p} / \sin \left(\frac{n \pi}{2}\right)
$$

and transformed by the factor of $\left(\cos \left(\frac{n \pi}{2}\right)-i \sin \left(\frac{n \pi}{2}\right)\right)$, which is drawn in red $(\mathrm{c},-)$. The curve is a perfect semi-circle extending the depressed semi-circle. With eq (8) plugged into eq (7), the effective capacitance can be obtained as below,

$$
C_{\text {eff }}=\frac{\left(Y_{0} R_{p}\right)^{1 / n}}{R_{p}} \sin \left(\frac{n \pi}{2}\right)
$$

The physical meaning of the rotation is not yet clearly explored as the physical meaning of $n$ of a CPE is still controversial. But the rotation of the semi-circle can be comparable to depression of the semi-circle. A full rotation corresponds to no depression with $n=1$ while partial rotations do to partial depressions of the semi-circle with $n$ less than 1 .

\section{Results and Discussion}

Simulations for impedance spectrum are carried out for the three equivalent circuits in Fig. 1 with converted values obtained by the methods of Westing [10], Hsu [11] and this report; the simulation conditions are $R_{\mathrm{s}}=1 \Omega, R_{\mathrm{p}}=1 \times 10^{4} \Omega, Y_{0}=1 \times 10^{-5} \mathrm{~s}^{n} / \Omega$ and $n=0.8$ to 1 . In order to compare the methods, we should discuss about two observations on the imaginary number of the impedance $\left(-Z^{\prime \prime}\right)$ vs. $\log$ (fre-

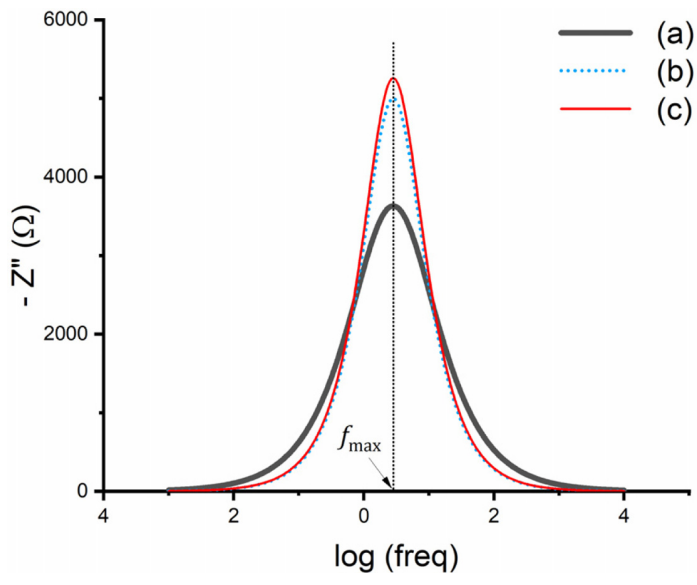

Fig. 3. Imaginary part of impedance spectra are plotted against frequency: $-Z^{\prime \prime} v s$. $\log$ (frequency). Curve (a) is taken from the Nyquist plot of Fig. 2(a). Curve (b) is from the impedance spectrum calculated with $R_{\mathrm{p}}$ and $C$ which is obtained by the method of C. H. Hsu and F. Mansfeld, eq (3). Curve (c) is from the impedance spectrum calculated with $R_{\text {eff }}$ and $C_{\text {eff }}$ which are obtained by the newly suggested method, eq (8) and (9). $f_{\max }$ is the frequency where $-Z^{\prime \prime}$ is the maximum.

Table 1. Comparison of the capacitances calculated by the methods of eq(2), eq(3) and this report for the circuit in Fig. 1(a) with a serial resistor. The simulation conditions: $R_{s}=1 \Omega$, $R_{p}=1 \times 10^{4} \Omega, Y_{0}=1 \times 10^{-5} \mathrm{~s}^{n} / \Omega$ and $n=0.8$ to 1 .

\begin{tabular}{cccc}
\hline \hline $\mathrm{n}$ & \multicolumn{3}{c}{ Calculated Capacitance $\left(\mathrm{F} / \mathrm{cm}^{2}\right)$} \\
\cline { 2 - 4 } & eq $(2)$ & eq $(3)$ & new \\
\hline 1 & $1.00 \times 10^{-5}$ & $1.00 \times 10^{-5}$ & $1.00 \times 10^{-5}$ \\
0.95 & $0.89 \times 10^{-5}$ & $0.89 \times 10^{-5}$ & $0.88 \times 10^{-5}$ \\
0.9 & $0.78 \times 10^{-5}$ & $0.77 \times 10^{-5}$ & $0.76 \times 10^{-5}$ \\
0.85 & $0.69 \times 10^{-5}$ & $0.67 \times 10^{-5}$ & $0.65 \times 10^{-5}$ \\
0.8 & $0.59 \times 10^{-5}$ & $0.56 \times 10^{-5}$ & $0.54 \times 10^{-5}$ \\
\hline
\end{tabular}

quency) shown in Fig. 3. One is that all the maximum numbers are found at the same frequencies, $f_{\max }$, which confirms the equivalency of the circuits as shown by the results in the literature [11]. The other one is that the values are different along the frequency, which is explained in terms of resistance. The integration method enables to theoretically calculate the resistance using the following equation [13],

$$
R=\left(\frac{9.2}{\pi}\right)\left(\int_{\infty}^{f_{\max }} Z^{\prime \prime}\right) d \log f
$$

and the calculation result exactly matches to $R_{\text {eff }}$, 
which also confirms that a parallel connection of a CPE and a $R_{\mathrm{p}}$ can be described as that of a $C_{\text {eff }}$ and a

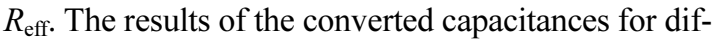
ferent $n$ values are summarized in Table 1, and compared by methods using eq (2) and eq (3). The values are learned to be nearly the same within tolerance of $3 \%$ for $n=0.9$ corresponding to a usual case and even $9 \%$ for $n=0.8$ corresponding to extreme cases.

\section{Conclusions}

In analysis of electrochemical impedance spectra, the capacitive component is usually observed to have a non-ideal behavior and described as a constant phase element (CPE) of which the parameters are $Y_{0}$ and $n$ instead of the conventional capacitance, $C$. While capacitance is clearly defined to have physical meaning, $Y_{0}$ is ambiguously known. Nevertheless, it is still considered to be analogous to capacitance, and has been studied to have a physical quantity equivalent to capacitance. To that purpose, two formula are already suggested and used widely. On the extension of the development, in this report, another formula is suggested with a different approach. In this approach, a decaying current generated by a CPE and a resistor is equivalently described as the one by an effective capacitor and an effective resistor, so that the current should have a time-constant. The time constant is related to $Y_{0}$ and $n$ which lead to the values of the effective capacitor and resistor simultaneously. While the previous two methods only consider $Y_{0}$ and $n$ of the CPE, the new one takes the effective resistance into account for the conversion process.

Among the three formulae suggested, which will give the most accurate result? The choice does not have to be worried about because there does not exist the exact value. A CPE holds both nature of capacitance and resistance which are dependent on frequencies, and the values are only estimated equivalently based on conditional assumptions. As a matter of fact, the values obtained by those methods are as slightly different as no significance is considered in the practical measurements. Any formula can be chosen depending on the conditions of the system of interest.

\section{Acknowledgment}

This work was supported by a Research Grant of Pukyong National University(2019).

\section{References}

[1] B.-Y. Chang and S.-M. Park, Annu. Rev. Anal. Chem, 2010, 3, 207-229.

[2] J.-B. Jorcin, M.E. Orazem, N. Pébère and B. Tribollet, Electrochim. Acta, 2006, 51(8-9), 1473-1479.

[3] A. Lasia, in: Conway, B. E. and Bockris, J. O. M., Electrochemical Impedance Spectroscopy and its Applications in Modern Aspects of Electrochemistry, Springer US, 2002.

[4] E. Barsoukov and J.R. Macdonald, Impedance Spectroscopy: Theory, Experiment, and Applications, John Wiley \& Sons, 2005.

[5] P. Zoltowski, J. Electroanal. Chem, 1998, 443(1), 149154.

[6] E. Cuervo-Reyes, C.P. Scheller, M. Held and U. Sennhauser, J. Electrochem. Soc, 2015, 162(8), A1585A1591.

[7] M. Naseri, L. Fotouhi and A. Ehsani, J. Electrochem. Sci. Technol, 2018, 9, 28-36.

[8] F. Fekri, M. Shahidi, M.M. Foroughi and M. Kazemipour, J. Electrochem. Sci. Technol, 2019, 10, 148-158.

[9] W. Choi, H.-C. Shin, J.M. Kim, J.-Y. Choi and W.-S. Yoon, J. Electrochem. Sci. Technol, 2020, 11, 1-13.

[10] E.P.M. van Westing, G.M. Ferrari and J.H.W. de Wit, Corrosion Science, 1993, 34(9), 1511-1530.

[11] C.H. Hsu and F. Mansfeld, Corrosion, 2001, 57(9), 747748.

[12] M.E. Orazem, P. Shukla and M.A. Membrino, Electrochim. Acta, 2002, 47(13-14), 2027-2034.

[13] M. Kendig and F. Mansfeld, Corrosion, 1983, 39(11), 466-467. 